\title{
Control of a Mach reflection-induced interaction using an array of vane-type vortex generators
}

\author{
S. B. Verma ${ }^{1}$ - C. Manisankar ${ }^{1}$
}

Received: 28 June 2017 / Revised: 6 October 2017 / Accepted: 3 November 2017 / Published online: 24 November 2017

(C) Springer-Verlag GmbH Germany, part of Springer Nature 2017

\begin{abstract}
An experimental investigation was conducted to control a Mach reflection (MR)-induced flow separation in a Mach 2.05 flow using a $18^{\circ}$ shock generator (SG). The study was extended to four SG exit heights $(g / w)$ of $0.87,0.81$, 0.725 , and 0.66 primarily to study its effect on the extent of flow separation as well as on Mach stem height, with and without control. Two vane-type vortex generator configurations, namely the ramp vane (RV) with device heights $h / \delta=0.3,0.5,0.8$, and 1.0 and the rectangular vane (RRV) with $h / \delta=0.3$ and 0.5 , were studied for control. Each control device array was implemented $10 \delta$ upstream of the separation location for no control. For stable MR interactions (i.e., $g / w=0.87,0.81$ ), the extent of separation and the reattachment shock strength are seen to decrease with increase in $\mathrm{RV}$ height (with $h / \delta=1.0$ device showing $17 \%$ reduction). However, for unstable MR condition (i.e., $g / w=0.725$ ), RV devices of $h / \delta=0.8$ and 1.0 become ineffective. The RRV2 device $(h / \delta=0.5)$, on the other hand, was found to be more effective in reducing the extent of separation in both the stable (31\%) and unstable (24\%) MR conditions. The effectiveness of each control device is also accompanied with an increase in height of the Mach stem. This is, however, not seen as a serious limitation since in such strong interactions it is more important to prevent or avert an intake unstart condition. The separation shock unsteadiness or the $\sigma_{\max } / P_{\mathrm{w}}$ value, on the
\end{abstract}

Communicated by C.-Y. Wen and A. Higgins.

$凶$ S. B. Verma

sbverma@nal.res.in

C. Manisankar

manisankar@nal.res.in

1 Experimental Aerodynamics Division, National Aerospace Laboratories, Council of Scientific and Industrial Research (CSIR), Bangalore 560017, India other hand, is seen to increase considerably with controls and seems to be almost independent for $h / \delta \geq 0.5$.

Keywords Mach reflection - Flow separation - Mach stem · Shock strength · Vane-type control device

\section{Introduction}

In supersonic intakes, the oncoming air is compressed through a system of oblique shock waves and is finally decelerated to subsonic velocities through a near-normal terminating shock, Fig. 1a. The upstream Mach number to such near-normal terminating shocks may range from 1.3 to 2 depending on the constraints imposed by the overall system size that limits the compression achievable through these series of oblique shocks [1]. Not only do such strong interactions increase the stagnation pressure loss that affects the inlet performance but can also cause large areas of flow separation upstream of the subsonic diffuser, thereby introducing flow distortions that degrade the engine performance. At certain off-design operating conditions, the situation can further worsen causing the terminating shock to travel upstream, leading to intake unstart. Additionally, the shock unsteadiness associated with such interactions [2,3] can contribute toward fatigue failure in supersonic inlets [4] and introduce dynamic loads on the engine [1]. As a result, it is imperative to use flow control techniques so as to alleviate or diminish the adverse effects of such interactions and the problems associated with them. Figure $1 \mathrm{~b}$ shows the schematic of a Mach reflection (MR or Edney type II interference)-induced interaction initiated by an incident shock. As can be seen, a near-normal shock, or a Mach stem, is formed between the two triple points TP1 and TP2, the height $\left(H_{\mathrm{m}}\right)$ of which is controlled by the strength of the interaction. It would be 
Fig. 1 Schematics showing a the SWBLI in supersonic intakes and $\mathbf{b}$ important flow features MR-induced shock-wave/boundary-layer interaction. $X_{\text {imp }}$-incident shock impingement location, $X_{\mathrm{s}}$-separation location
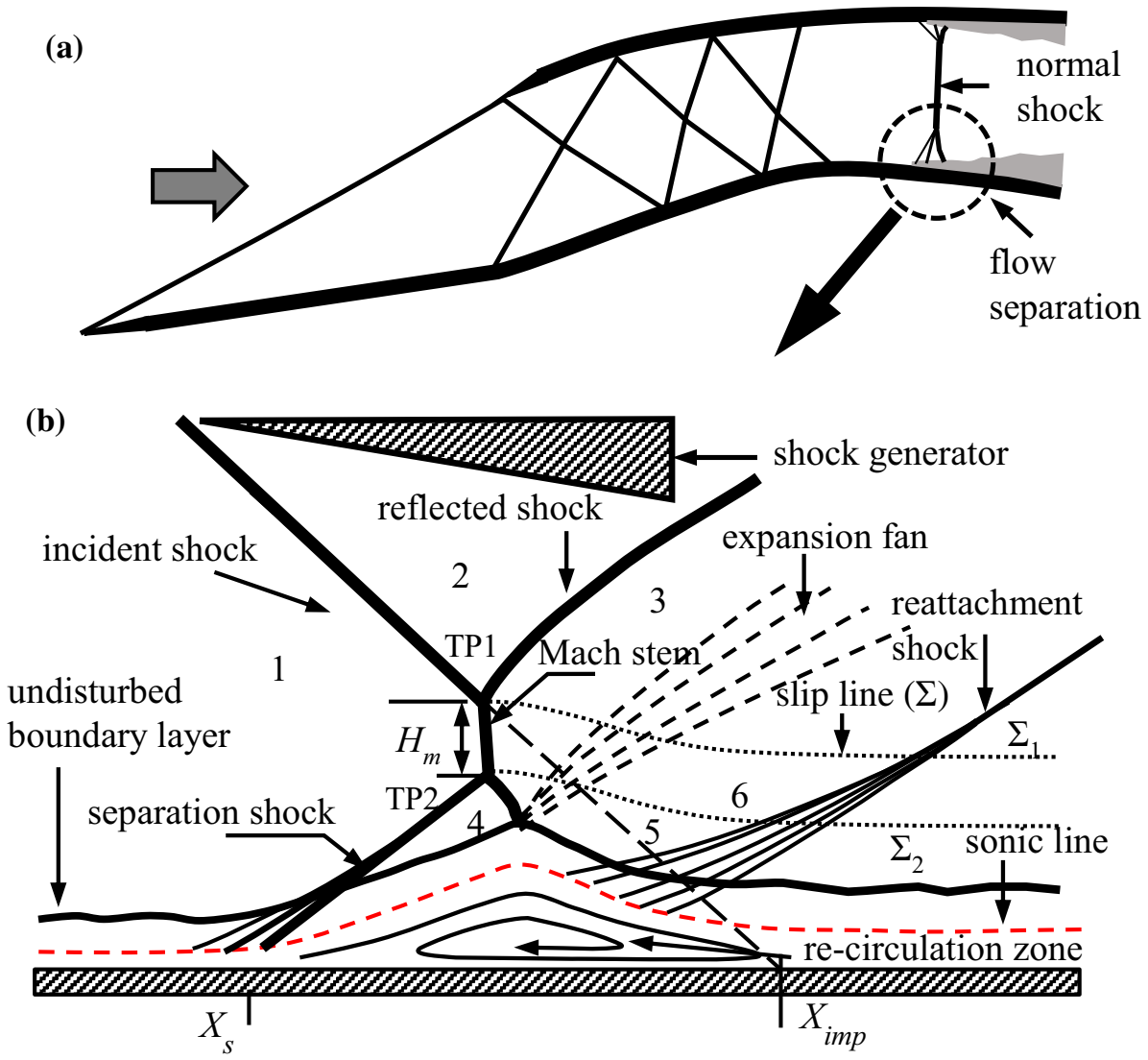

worthwhile to note that while the separation bubble in such interactions presents itself as an aerodynamic blockage to the oncoming flow, the presence of strong shocks, on the other hand, results in loss of pressure recovery and adds to the wave drag. The idea of modifying or controlling such an interaction therefore relies on minimizing or mitigating these adverse effects for enhanced performance.

Flow control techniques are broadly categorized into two classes, namely shock control and boundary-layer manipulation. The former approach uses techniques to weaken the near-normal shock by splitting it into weaker shocks such as a lambda shock structure pattern primarily to reduce the total pressure losses and hence the wave drag. These include use of porous cavities [5] and shock control bumps (SCB) (two-dimensional [5,6] or three-dimensional [7-11] in configuration). The latter, on the other hand, works toward adding momentum to the near-wall region to energize the boundary layer so that it is able to sustain relatively higher adverse pressure gradients and hence delay or avoid separation. Various methods to achieve this include blowing $[12,13]$, suction $[14,15]$ or natural bleed [16], and vortex generator (VG) devices [17-22]. The VG devices maybe further subdivided into active or passive based on the approach used. For example, synthetic jets [23-25], plasma jets [26$28]$, steady jets [29-31], and pulsed jets $[26,27,32]$ fall into the category of active VG devices, while mechanical VG devices such as vane-type [19,21,22,33-40] and delta ramps [17-22,36,38-40] come under the passive control category. The working principle of these devices is through the generation of streamwise vortices (co- or counter-rotating) to improve the overall structure of the incoming boundary layer. A detailed comprehensive view of the various control techniques, both active and passive, that have been used to control supersonic shock-wave/boundary-layer interactions (SWBLI) can be found in [41]. Past studies also report that although the active devices have the added flexibility to switch on and off on-demand [29], suppress specific flow frequencies [41] and reduce device drag when not in use, the system cost and the complexities associated with them makes it difficult to implement and maintain [22]. The mechanical devices, on the other hand, are low cost, simple, and rugged in design $[36,37,42]$ making them potential candidates for use as flow control devices in air intakes. However, the parasitic drag associated with them can be a disadvantage [43]. From this perspective, the sub-boundary layer or micro-VGs $(h / \delta<1)$ are preferable. The primary advantage of these micro-VGs is that the vortices generated from them remain in the boundary layer over larger streamwise distances improving their effectiveness compared to the conventional VGs $(h / \delta>1)$. More recent studies have further revealed [36,37] 
(a)

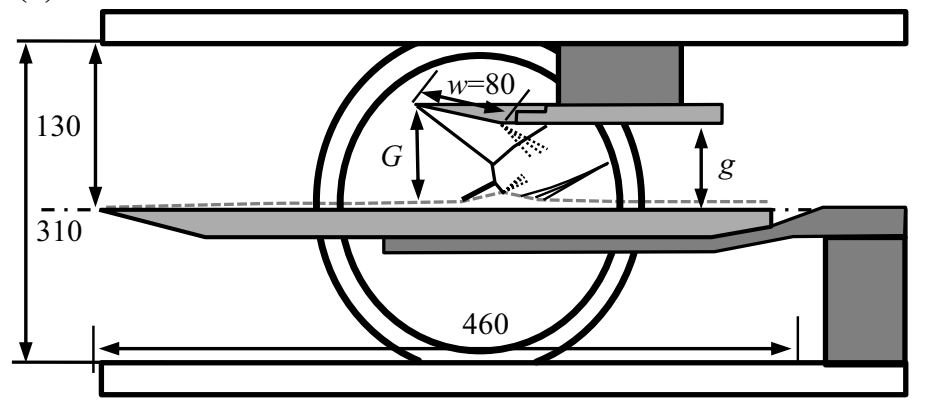

(b)

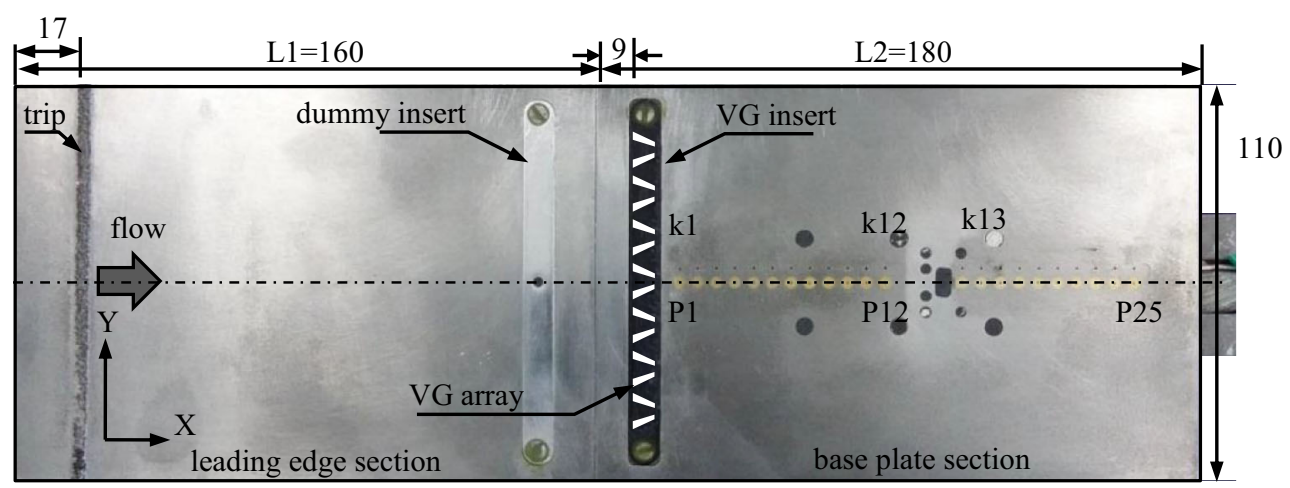

Fig. 2 Test model details a schematic of the experimental setup and $\mathbf{b}$ flat-plate model details with the pressure sensors and VG insert locations. All dimensions are in $\mathrm{mm}$

that for a given device configuration the overall flow features and the momentum added to near-wall region scales linearly with device height. This means that a larger-sized control device is more effective in stabilizing the interaction than a smaller one.

Most of the previous studies using mechanical VGs have been either in the subsonic $[19,21,35]$ or transonic $[5,17,22$, $36,37]$ regimes. A few experimental studies have also been reported in supersonic interactions but mostly using microramps of Anderson configuration [18,20,42,44-46]. More recently, an experimental study has been reported by Verma and Manisankar [38] to assess and compare the effectiveness of various VG configurations in controlling a regular reflection (RR)-induced interaction. With regard to control of normal shock-induced interactions, most of the earlier reported computational [36,37] and experimental [39,40] studies have been in the transonic regime. These studies were conducted to control the near-normal shock-induced interactions with relevance to flow conditions on transonic wings and those prevalent upstream of the diffuser section in intakes. With regard to the latter, the Mach numbers encountered are higher and generally in the range of 1.3-2.0 [1]. It was therefore decided to study the control of a Mach reflection (MR)-induced separation at Mach 2.0. A similar such study [47] was earlier reported by the present authors but for various VG devices of $h / \delta=0.3$ and for only one non- dimensional shock generator exit height $g / w$ of 1.4. Here the dimension $g$ is the SG exit height and $w$ the SG ramp length, as shown in Fig. 2a. However, the height effect $(h / \delta)$ for the ramp vane (RV) devices and variation in $g / w$ was not studied. The latter approach helps to study the beginning or initial stages of intake unstart conditions wherein the extent of the separation bubble begins to increase with the separation point moving rapidly forward. Keeping this in view, the objective of the present investigation was focussed (i) to study the effect of four $g / w$ values $(0.87,0.81,0.725$, 0.66 ) on the extent of flow separation as well as on Mach stem height and (ii) to attempt controlling the above interactions using two vane-type vortex generator configurations, namely the RV and the rectangular vane (RRV) type. These objectives were primarily chosen with the intention to study whether the control effectiveness at higher $g / w$ values will still hold in much stronger interactions as well.

\section{Experimental setup and procedure}

\subsection{Wind tunnel facility and model details}

An experimental investigation was conducted in the $0.46 \mathrm{~m} \times 0.31-\mathrm{m}$ blowdown trisonic wind tunnel at the National Aerospace Laboratories (NAL) in India to study 

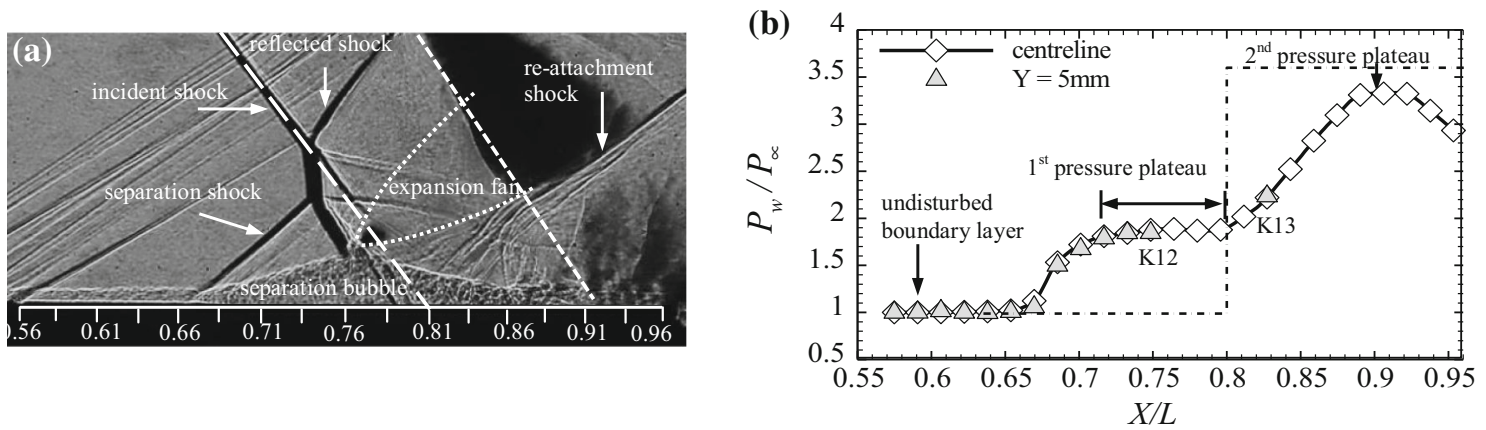

Fig. 3 Plot showing a the schlieren image of the Mach reflection-induced interaction and $\mathbf{b}$ the associated streamwise mean pressure distribution; no control, $X / \delta=10, g / w=0.81$

control of Mach reflection-induced boundary-layer interaction using vane-type VG devices. The freestream Mach number and the unit Reynolds number $(\mathrm{Re} / L)$ for all the tests was $2.05 \pm 0.02\left(U_{\infty}=523 \mathrm{~m} / \mathrm{s}\right)$ and $25 \times 10^{6} \mathrm{~m}^{-1}$, respectively. For all the tests, the wind tunnel operating stagnation pressure $\left(P_{0}\right)$ was within the range of $208.5 \mathrm{kPa} \pm 2 \%$ (absolute) and for temperature $\left(T_{0}\right)$ within $298 \mathrm{~K} \pm 0.4 \%$. The wall temperature was assumed adiabatic, and the turbulence levels in the tunnel were approximately $0.2 \%(\%$ $C_{\text {prms }}$ ). The test model was a sting-supported flat-plate setup mounted along the tunnel centerline as shown in Fig. 2a. An $18^{\circ}$ shock generator (SG) wedge of $220 \mathrm{~mm}$ width $(2 b)$ and SG ramp length $(w)$ of $80 \mathrm{~mm}$ was mounted on the tunnel top wall to generate a shock wave $\left(\beta=49.78^{\circ}\right)$ that impinged on the flat plate below to initiate the MR interaction, Fig. 2a. The Reynolds number $\left(\operatorname{Re}_{x}\right)$ based on the flat-plate length up to the VG insert was $4.26 \times 10^{6}$. A boundary-layer trip made of 60-grit carborundum particles and of length $4 \mathrm{~mm}$ was placed $17 \mathrm{~mm}$ from the leading edge to ensure a turbulent boundary layer. For mean pressure $\left(P_{\mathrm{w}}\right)$ measurements using electronic pressure scanners (ESP), a total of 25 ports (P1-P25) are available along the plate centerline to capture the entire interaction. However, for unsteady pressure measurements, only 13 Kulite pressure transducers have been provided primarily to capture the flow details in the vicinity of the separation region. As a consequence of this, the rms values defined as $\sigma=\sqrt{\frac{\sum_{i=0}^{n}\left(P_{\mathrm{w}_{i}}-P_{\mathrm{w}}\right)^{2}}{(n-1)}}$ are available only for these locations. It may also be noted that these pressure ports are located $5 \mathrm{~mm}$ off the centerline, as shown in Fig. $2 \mathrm{~b}$, which corresponds to device off-center location of almost $100 \%$ span.

With the present setup, independent tests were conducted for four $g / w$ values of $0.87,0.81,0.725$, and 0.66 . This corresponds to an inlet aspect ratio $(b / G)$ range of 1.22-1.5, respectively, which is free from wedge three-dimensional effects [48]. Here, $b$ is the spanwise half-width of the SG and $G$ is the SG inlet height (Fig. 2b). Figure 3a, b shows the schlieren image for a no-control case with $g / w=0.81$ and its associated mean pressure distribution, respectively. The inviscid pressure ratio for this interaction is 3.6. It can be seen that despite the difference in their spanwise locations, the Kulite and ESP pressure distributions show a similar pressure rise across the interaction on either side of the centerline indicating a straight separation line in spanwise direction. However, due to the finite spanwise extent of the flat plate, three-dimensional effects do influence the interaction but only toward the outer edges of the flat plate. Keeping this in mind, it may be emphasized that the discussion of the results in this paper will be limited only to that finite region of the flow about the centerline where the separation line was seen to be straight in the surface oil pictures (not shown) for no control. It may further be noted that no side fences were used on the plate sides in order to facilitate schlieren imaging.

Two types of control device configurations, namely an array of the RV and RRV, were investigated (Fig. 4). Table 1 shows the designations used for the control devices in the present paper based on their configuration and device height. For both the VG configurations, an inter-device spacing (center-to-center) of $s=12 \mathrm{~mm}$ and an inter-vane spacing of $1 h$ is maintained for all device heights resulting in an array of seven control devices for each configuration. The middle VG for each array was centered on $Y=0 \mathrm{~mm}$ (Fig. 2b). The boundary-layer thickness $(\delta)$ as estimated from the schlieren images was found to be approximately $3.4 \pm 0.04 \mathrm{~mm}$ just upstream of the separation for no control. The boundary-layer thickness was also estimated based on length Reynolds number $\operatorname{Re}_{x}$ for turbulent flows $(3.48 \mathrm{~mm})$ with a corrected value of $(3.56 \mathrm{~mm})$ for compressible flows as suggested by Van Driest [49]. However, such an estimate may differ from the boundary-layer thickness calculated from velocity profile by $3 \%$ [50]. For no control, the mean skin friction coefficient $c_{\mathrm{f}}$, after correction for turbulent flows [49], is $2.8 \times 10^{-3}$ and the shape factor is 1.3 . While the ramped triangular vane was studied for various device heights $h / \delta=0.3,0.5,0.8$, and 1.0 , respectively, the rectangular vane device was tested for only $h / \delta=0.3$ and 0.5 . An array of each of these control devices was implemented at $10 \delta$ upstream of the separa- 
Fig. 4 Schematics of the vane-type control configurations. a Ramp vane $(h / \delta=0.3,0.5,0.8,1.0)$ and b rectangular vane $(h / \delta=0.5)$
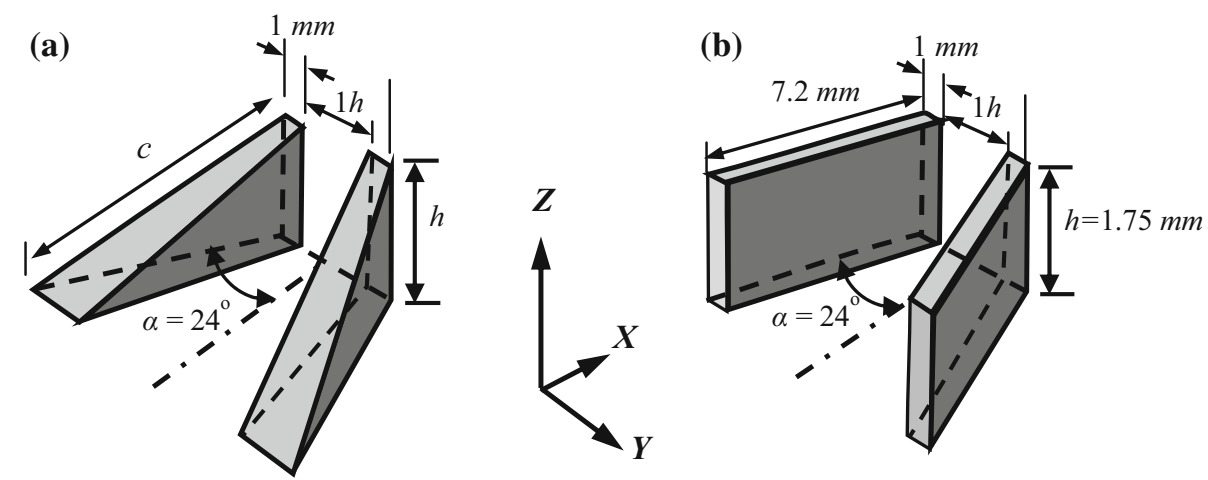

Table 1 Details and designations for each control device pair

\begin{tabular}{lllll}
\hline $\begin{array}{l}\text { Control } \\
\text { device }\end{array}$ & $\begin{array}{l}\text { Height } \\
(h \text { in mm })\end{array}$ & $h / \delta$ & $\begin{array}{l}\text { Angle of } \\
\text { attack } \alpha\left(^{\circ}\right)\end{array}$ & $\begin{array}{l}\text { Projected } \\
\text { area }\left(\mathrm{mm}^{2}\right)\end{array}$ \\
\hline RV1 & 1.0 & 0.3 & 24 & 2.93 \\
RV2 & 1.7 & 0.5 & 24 & 4.98 \\
RV3 & 2.7 & 0.8 & 24 & 7.96 \\
RV4 & 3.4 & 1.0 & 24 & 9.96 \\
RRV1 & 1.0 & 0.3 & 24 & 4.98 \\
RRV2 & 1.7 & 0.5 & 24 & 9.96 \\
\hline
\end{tabular}

tion location for no control. Both off- and on-surface flow visualizations were undertaken. Still schlieren images of the flowfield were obtained using a Z-type schlieren setup with a vertical knife arrangement and using a Nikon D1x digital SLR camera with a 300-mm lens and exposure time of $125 \mu \mathrm{s}$. The setup used 3.0-m-focal-length spherical mirrors to collimate and refocus the illumination source at the knifeedge location. A Palflash 501 was used as the light source with spark duration set at $100 \mu$ s. Surface flow topologies with and without control were studied using the conventional surface oil pigment mixture comprised of titanium dioxide powder, vacuum pump oil, and oleic acid.

\subsection{Experimental accuracy}

The tunnel stagnation pressure, $P_{o}$, was acquired using a DRUCK 4010 series pressure transducer of $1379 \mathrm{kPa}$ range with $\pm 0.1 \%$ of full-scale accuracy, while the static pressure measurements such as $p_{\mathrm{w}}$ or surface wall pressure and $p_{\infty}$ or freestream pressure were acquired using ESP scanners of $206.8 \mathrm{kPa}$ range with $\pm 0.04 \%$ of full-scale accuracy. The pressure transducers were calibrated using a five-point calibration procedure before the start of the experiments. Further, a single-point check calibration was performed each day to check for any drift in error. The uncertainties in the pressure measurements were estimated using a statistical approach based on repeatability tests. The estimated uncertainty in measurement of total pressure was $\pm 1.4 \mathrm{kPa}$ and that for static pressure measurements was $\pm 0.7 \mathrm{kPa}$. The Kulite transducers for unsteady pressure measurements were calibrated statically, and the uncertainty obtained from calibration was found to be within $\pm 1 \%$ of full scale. However, in the intermittent region of separation that is associated with high levels of flow unsteadiness, the average pressure uncertainty is likely to be somewhat greater. The repeatability of the rms values in the interaction region was found to be roughly within \pm 0.04 .

\subsection{Signal conditioning and data acquisition system}

The mean static pressure on the flat plate was measured using Pressure Systems ESP-16HD 16-port scanners. These scanners were calibrated in situ using a Druck calibrator Model DPI-610. An eight-channel signal conditioner module (SCXI-1520) from National Instruments was used for acquisition of the analog signals from the pressure scanners. The analog signals were then digitized using a 16-channel 16bit A/D card (NI-6036) that has a maximum sampling rate of $200 \mathrm{kS} / \mathrm{s}$. The present data were acquired at $500 \mathrm{~Hz}$, with 500 samples taken for each port location. This resulted in an averaging time of $1 \mathrm{~s}$. The unsteady pressure data were measured using 13 fast piezo-resistive Kulite model XCQ-093 Mscreen (pressure range $170 \mathrm{kPa}$ ) transducers. The presence of the protective screen limited the frequency response of these transducers to $50 \mathrm{kHz}$. The Kulite transducers had a pressure sensitive area of $0.071 \mathrm{~cm}$ and an outer casing diameter of $0.26 \mathrm{~cm}$. The transducers were not flush mounted on the base plate. Instead, a small orifice (of $1 \mathrm{~mm}$ length and $0.5 \mathrm{~mm}$ diameter) connected the transducer to the flow. According to the manufacturer's specifications, these transducers have a 
natural frequency of approximately $250 \mathrm{kHz}$. The sensitivity of the transducers is typically $0.4-0.6 \mathrm{mV} / \mathrm{kPa}$. These transducers were calibrated statically. The transducer data were acquired using truly simultaneous acquisition card NI4495 DC series (with 24-bit resolution) at a sampling frequency of $50 \mathrm{kHz}$. Each sensor was powered by DC power supply, and the signal was passed through an amplifier and a signal conditioner. A low-pass filter of $20 \mathrm{kHz}$ was applied post-acquisition during data processing. For each transducer channel, 200 records of 4096 were acquired yielding a total of 819,200 data points per channel per tunnel run.

\section{Results and discussion}

\subsection{No control}

Independent tests were initially conducted for no-control case for four SG exit heights or $g / w$ values. To achieve this, each time the shock generator was lowered to reduce the $g / w$ value, and it was appropriately moved back so as to ensure that the separation location remains the same, i.e., $X / \delta=10$. Figure 5 shows the set of schlieren images for the three $g / w$ values of $0.87,0.81$, and 0.725 , respectively. Figure 6 on the other hand shows the corresponding mean pressure distributions and the associated shock polars. The flow conditions on the shock polar represented by numbers $1-5$ are shown in Fig. 1b. The non-dimensional Mach stem height $\left(H_{\mathrm{m}} / w\right)$ and the shock strengths $\left(\Delta P / p_{1}\right.$ - separation shock, $\Delta P / p_{2}-$ reattachment shock) are extracted from Figs. 5 and 6 , respectively and plotted in Fig. 7a, b for comparison. Here, $H_{\mathrm{m}}$ is the Mach stem height (as defined in Fig. 1b) and $\Delta P$ is the shock strength defined as $\left(p_{2}-p_{1}\right)$ and $\left(p_{3}-p_{2}\right)$ for separation and reattachment shocks, respectively, as shown in Fig. 6a. The $\Delta S / w$ values, which correspond to the distance between the physical separation and reattachment locations, are extracted from the surface oil tests. While the strength of both the separation and reattachment shocks is seen to remain almost similar for $g / w$ of 0.87 and 0.81 , it shows a significant increase in its values with further decrease in $g / w$ (Fig. 7a). This indicates that the interaction becomes much stronger for $g / w$ values of 0.725 and 0.66 (as is also seen in the shock polars shown in Fig. 6b) which results in a significant increase in extent of separation. In Fig. 6b, the polar 1-2 represents the pressure rise $P / P_{\infty}$ experienced by an incident shock in a $M=2.05$ flow after undergoing a flow deflection of $18^{\circ}$. Point 2 on it marks the origin of the reflected shock polar 23-2. On the other hand, the polar 1-4 represents the pressure rise achieved through the separation shock and the resulting flow deflection experienced due to the separation bubble located at the origin of the reflected shock polar 4-5-4. The states 3, 6 and 5, 6 represent the flow condition downstream of each of the two triple points TP1 and TP2. The gap between
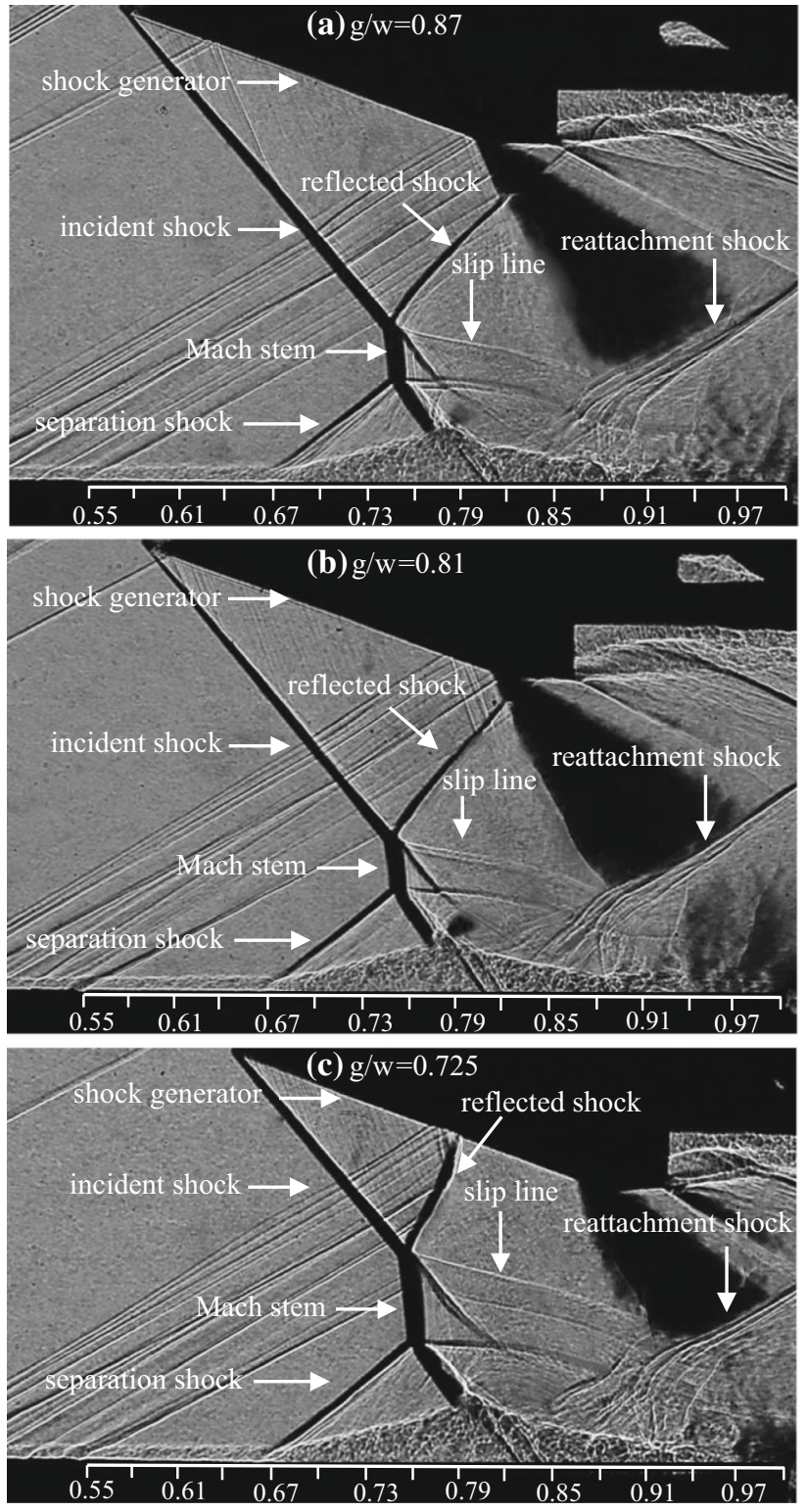

Fig. 5 Schlieren images showing the effect of variation in $g / w$ on the flow structure associated with Mach reflection in the present test setup

the states 5, 6 and 3, 6 represents a strong solution regime or the presence of a Mach stem. Figure $7 \mathrm{~b}$ also shows that there is only a small change in values of $H_{\mathrm{m}} / w$ and $\Delta S / w$ in going from $g / w$ of $0.87-0.81$. However, the increase in these values is significant for the interactions with $g / w$ of 0.725 (Fig. 5c) and 0.66 (schlieren image not shown) which shows the highest. The large increase in $H_{\mathrm{m}} / w$ and $\Delta S / w$ values simulates the beginning of the intake unstart condition wherein the separation location is driven upstream and out of the intake. It may, however, be pointed out that for $g / w$ of 0.66 , since the separation front moved significantly forward and up to the VG insert location, the control studies for it 
(a)

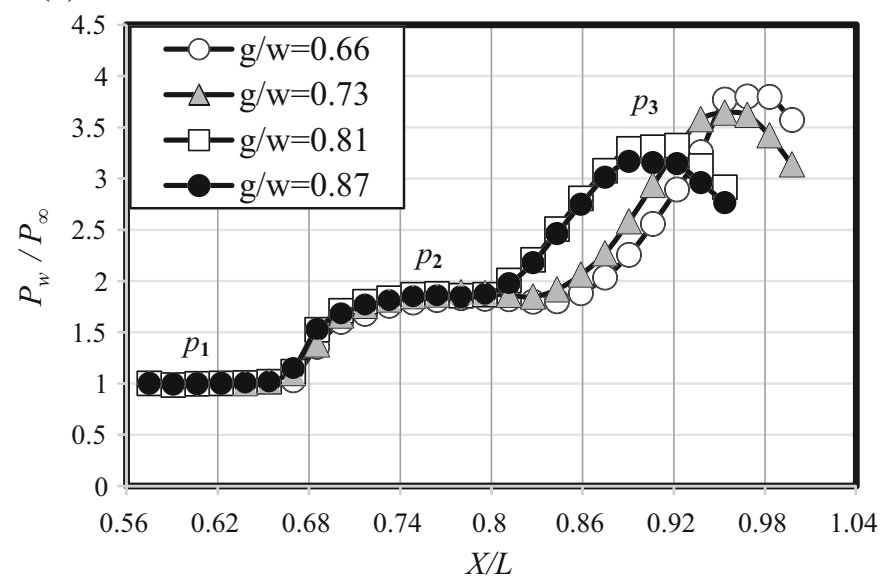

(b)

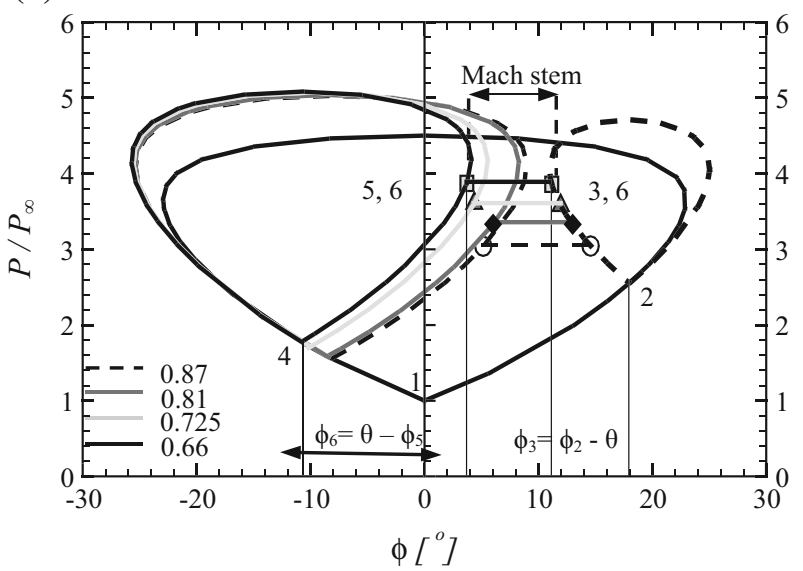

Fig. 6 Variation in a the streamwise mean pressure distribution and $\mathbf{b}$ the associated shock polars with choice of regions 1-6 as defined in Fig. 1b; no-control case for four $g / w$ values
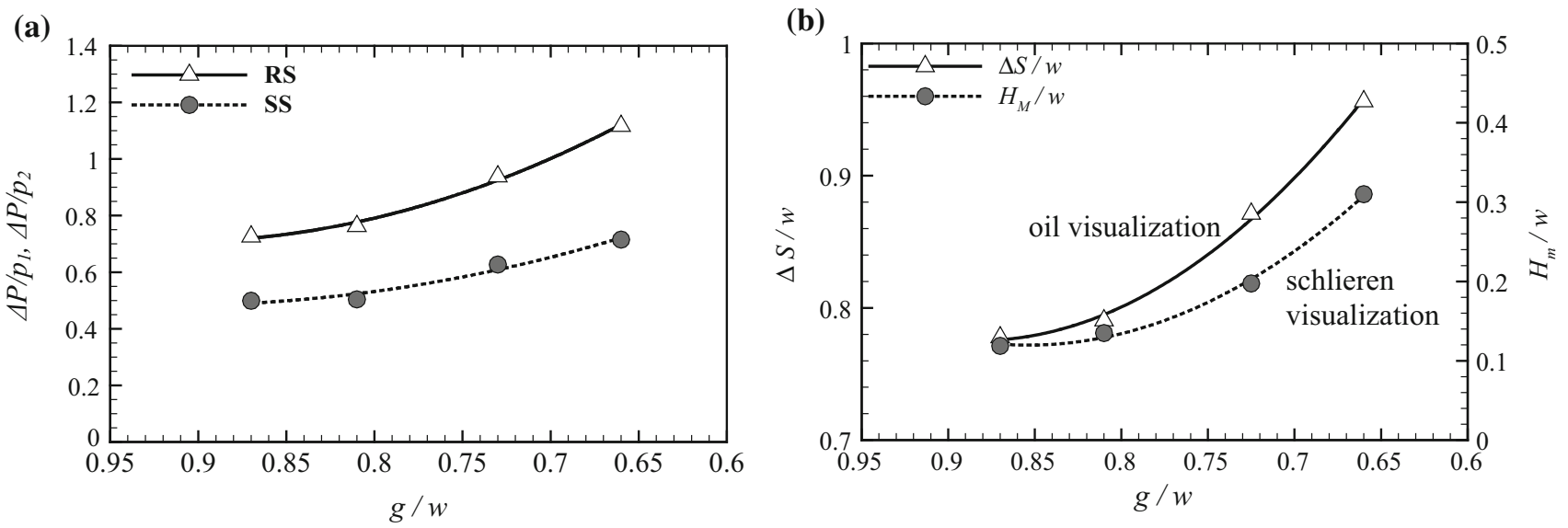

Fig. 7 Effect of variation in $g / w$ on a the separation shock (SS) and reattachment shock (RS) strengths and $\mathbf{b}$ the extent of separation bubble length and Mach stem height

were abandoned and conducted for only the remaining three $g / w$ values.

Earlier studies by Li and Ben-Dor [51] have shown that for such MR configurations, there exists a minimum value of $g / w$ at which the reflected shock wave begins to just graze the shock generator trailing edge, as shown in Fig. 5b. At this condition, the MR is considered to be stable [52]. Further lowering the $g / w$ value causes the reflected shock to impinge on the SG wedge surface wherein it reflects either as a RR or MR depending upon the local flow conditions, as shown in Fig. 5c. The MR in such conditions is termed unstable [52]. Further lowering the $g / w$ value causes the Mach stem to continue moving further upstream until, finally, the MR vanishes and a bow shock wave is established ahead of the leading edge of the reflecting wedge [51]. A careful observation of Fig. 5b, c clearly indicates that for the present test setup, the minimum $g / w$ value for a stable MR condition is approximately 0.81 . Similarly, $\mathrm{Li}$ and Ben-Dor [51] point out that there also exists an upper limit for which $g / w$ is so large that the leading characteristic of the expansion fan from the SG wedge trailing edge begins to intersect the incident shock. At such point, the expansion wave can influence both the strength and the orientation of the incident shock and hence the overall interaction.

\subsection{With control}

VG devices in the form of ramped vanes and rectangular vanes were implemented $10 \delta$ upstream of the separation location to control the interaction for test conditions with $g / w$ values of $0.87,0.81$, and 0.725 . Figure $8 \mathrm{a}, \mathrm{c}$, e shows the streamwise distribution of mean pressure distribution obtained using ESP along the plate centerline with and without control for each $g / w$ value. The plots on the right, Fig. 8 b, 
(a)

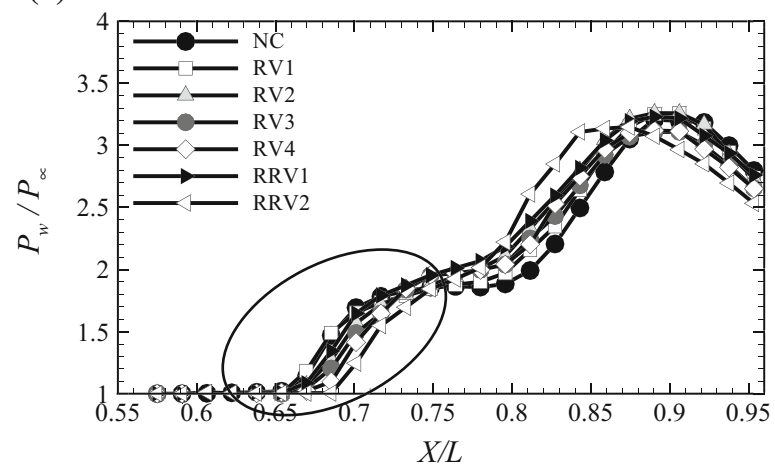

(c)

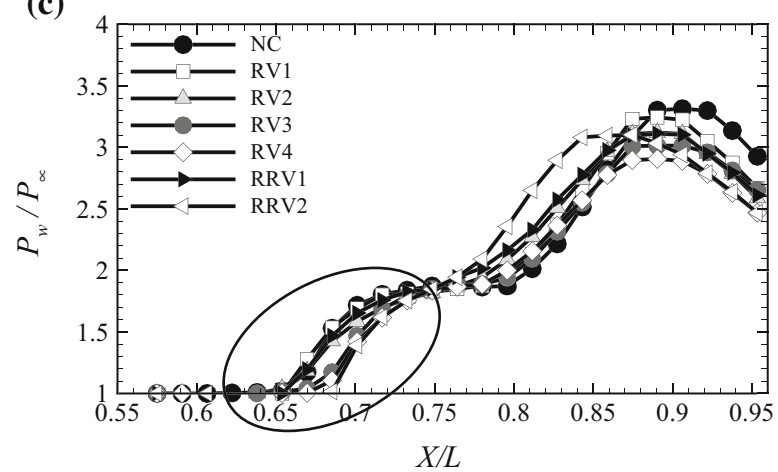

(e)

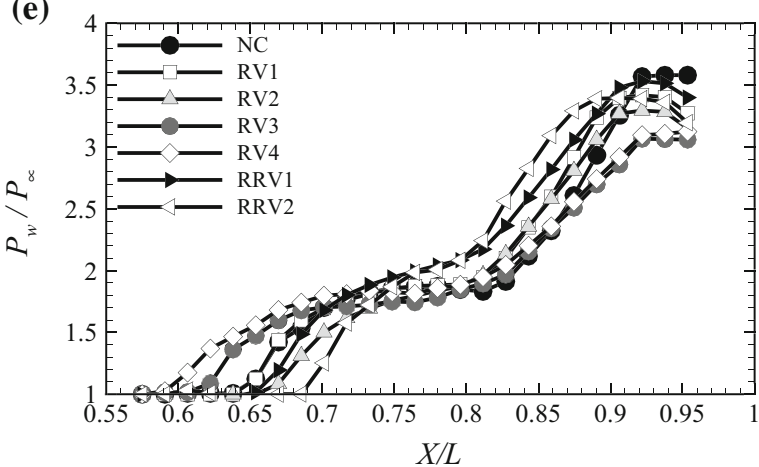

$X / L$ (b)

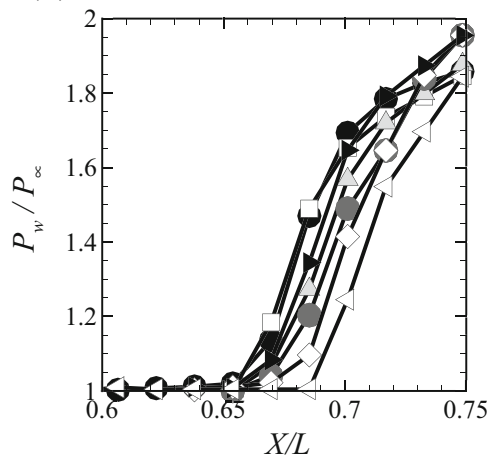

(d)

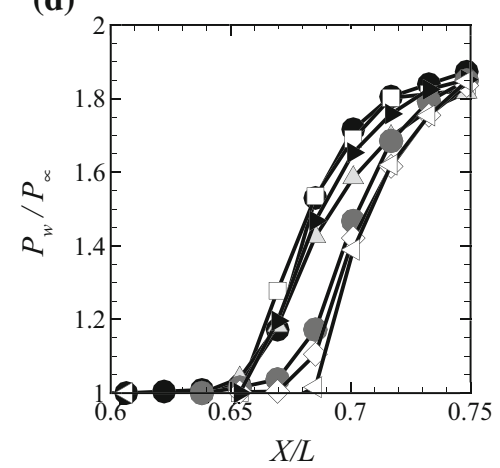

Fig. 8 Streamwise distribution of mean pressure with and without control for $\mathbf{a}, \mathbf{b} g / w=0.87$, $\mathbf{c}, \mathbf{d} g / w=0.81$, and $\mathbf{e} g / w=0.725$. $N C$ no-control case

d, show a zoom of the separation region marked by an ellipse in their respective plots, Fig. 8a, c, respectively, for clarity and discussion. It can be seen that for interactions such as those initiated by stable MRs, only the control devices with $h / \delta \geq 0.5$ are able to effectively reduce the extent of separation, Fig. 8a-d, and hence the overall interaction. From the perspective of pushing the separation location downstream, the most effective RV configuration is RV4, which is followed by RV3, RV2, and finally RV1 which shows similar separation location as that for no control. However, all the control devices show an upstream movement of the reattachment location or in the location of the second rise in wall pressure even for RV1. For RRV devices, on the other hand, the relevant change in separation and reattachment locations is much more significant compared to the RV devices of similar heights especially for RRV 2 case. In fact, the RRV2 device is seen to be even much more effective compared to RV3 and RV4 devices. Similar observations can be made for the interaction initiated with $g / w$ value of 0.81 . For the unstable MR case, i.e., $g / w=0.725$, Fig. 8e, other than the RV3 and RV4 devices which show a significant upstream movement of separation, the smaller height RV and RRV devices of $h / \delta=0.5$ are seen to initiate good control. The former is caused primarily due to very strong interaction shocks induced locally in the immediate vicinity upstream of the RV3 and RV4 devices. These shocks on interaction with the MR on the SG wedge surface modify it significantly causing the TP1 to move further upstream and hence the separation location that results in 
(a)

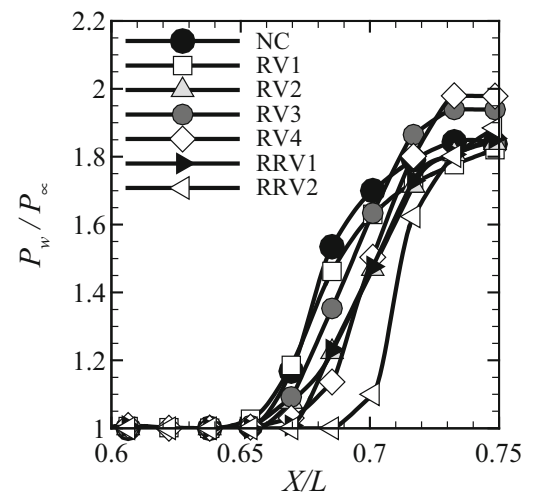

(b)

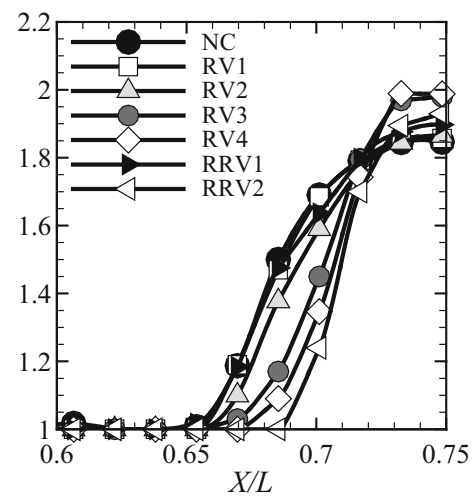

(c)

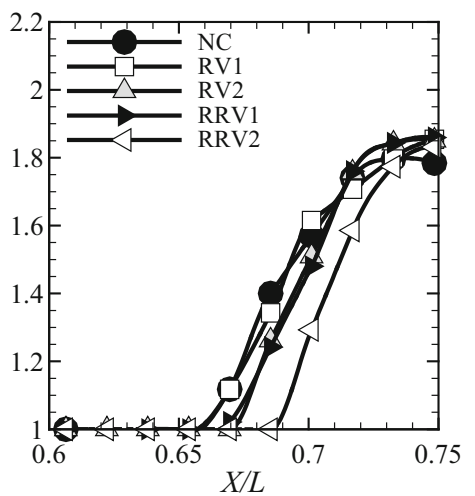

Fig. 9 Streamwise distribution of mean pressure from Kulite transducer locations with and without control for $\mathbf{a} g / w=0.87$, $\mathbf{b} g / w=0.81$, and $\mathbf{c} g / w=0.725$. $N C$ no-control case

(a)

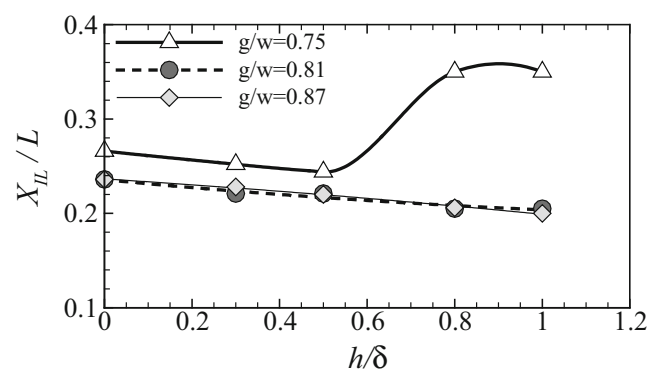

(b)

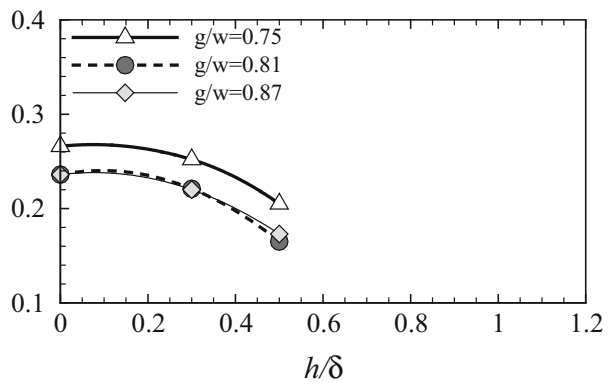

(b)

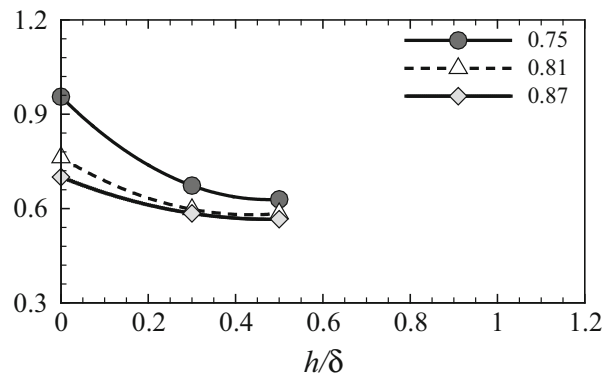

Fig. 11 Variation in the reattachment shock strengths for $\mathbf{a}$ RV and $\mathbf{b}$ RRV

a condition similar to flow blockage as during intake unstart. However, this is not so for devices with $h / \delta \leq 0.5$, which are still seen to be able to push the separation downstream and the associated reattachment location upstream relative to no control. Of the two types of device configurations, the rectangular vanes are once again seen to be much more effective compared to the ramped vanes of similar height. The data from the Kulite pressure sensors were also analyzed and are shown in Fig. 9. Comparing Figs. 8 and 9 show that although a significant three-dimensionality is introduced in the separation line with the implementation of controls, the RRV2 continues to show the most effective control capability compared to RV3 and RV4 under all test conditions.

The length of the interaction ( $X_{\mathrm{IL}} / L$, Fig. 1b), i.e., the distance from the first rise in wall pressure at separation to the beginning of the second pressure plateau (end of reattachment), and the reattachment shock strengths $\left(\Delta P / p_{2}\right)$ are extracted from these mean pressure plots, Fig. 8 , and plotted in Figs. 10 and 11, respectively. It can be seen that while the interaction length remains almost similar for $g / w$ values of 0.87 and 0.81 , the interaction length shows a gradual decrease in its value with increase in RV height, Fig. 10a, 
with RV4 showing maximum reduction of $17 \%$ relative to no control. A similar such observation can be made for $g / w$ value of 0.725 except for RV3 and RV4 for which the separation location is observed to move significantly upstream as discussed before. Comparing Fig. 10a, b clearly shows that the RRV2 device is able to significantly reduce the length of the interaction in both stable (by $31 \%$ ) and unstable (by 24\%) MR conditions. Similarly, for reattachment shock strength, a significant reduction in its value compared to no control is observed as the RV device height is increased, Fig. 11a, with RV4 showing maximum reduction of $23 \%$. In fact, it becomes almost similar for RV3 and RV4 indicating that increasing the device beyond $h / \delta$ of 0.8 has no effect. A reduction in the reattachment shock strength for RRV2 is observed to be approximately $20 \%$ for stable and $35 \%$ for unstable MR (Fig. 11b). It may, however, be noted that since VGs are known to generate CRVs that create regions of upwash and downwash as they develop downstream, a spanwise array of such VGs will result in a wavy or a corrugated separation line [38]. As a result, depending upon the placement of pressure transducers, the results will vary from the presently reported results which are valid for measurements made along the device centerline or the upwash region. However, it may be pointed out that the overall effectiveness of the control device will still be maintained irrespective of the measurements made in the region of upwash or downwash [38].

The rms $\left(\sigma / P_{\mathrm{w}}\right)$ values for each of the Kulite transducer locations were calculated. Figure $12 \mathrm{a}$ shows the distribution of streamwise rms values associated with the streamwise
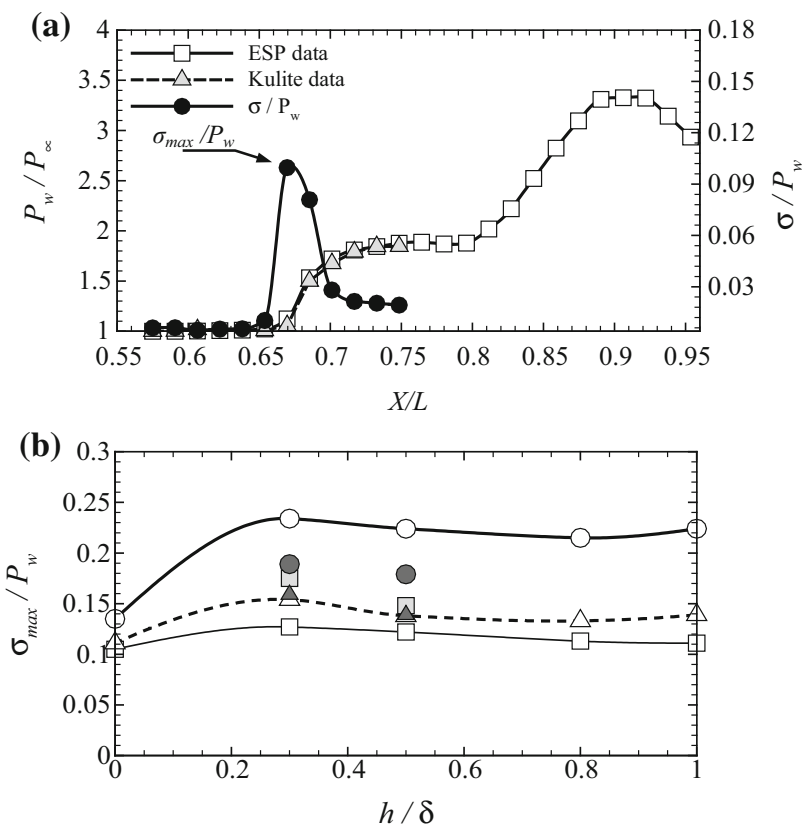

Fig. 12 Plots showing the variation in a streamwise distribution in $\mathrm{rms}$ values in the region of separation and $\mathbf{b}$ peak rms values with and without control for three $g / w$ values mean pressure distribution for no-control case and for $g / w$ value of 0.81 . It can be seen that the rms values show a significant increase in the region of first rise in wall pressure from its undisturbed boundary-layer value. In this region, the rms value reaches a peak $\left(\sigma_{\max } / P_{\mathrm{w}}\right)$, as shown in Fig. $12 \mathrm{a}$, after which its value begins to decrease as the plateau pressure region is gradually approached. In this region, it stabilizes to almost a constant value but continues to remain still higher than its value in the undisturbed boundary layer. The $\sigma_{\max } / P_{\mathrm{w}}$ values from all the test cases with and without control were extracted and are plotted in Fig. $12 \mathrm{~b}$ for comparison. It can be seen that for no control $(h / \delta=0$, Fig. 12b) the peak rms values show an increase with decrease in $g / w$ values which is primarily attributed to the increase in strength of the separation shock [2] as discussed earlier, Fig. 7a. With control, the peak rms value initially shows an increase for RV1 and thereafter shows an almost similar value independent of the device height. This trend remains the same with all $g / w$ values although a decrease in the rms values is seen with increase in $g / w$ value. However, the use of the RRV device brings down the peak rms values significantly though the values still remain higher than that observed for each no-control case. These and the previously discussed results indicate that while the length of interaction and the associated reattachment shock strength are considerably controlled using RV and RRV control devices with $h / \delta \geq 0.5$, the peak rms values in the intermittent region of separation show a significant increase compared to no control for each $g / w$ tested.

Figures 13 and 14 show the schlieren images of the interaction for the select test cases and for $g / w$ values of 0.725 and 0.81 , respectively. Only the images for the best performing control devices are shown for comparison relative to no control. For no control, upstream of separation, a series of weak waves (Mach waves with an approximate angle of $30^{\circ}$ ) are seen to emanate into the flow primarily from the front and base plate junction and the VG insert. These weak disturbances do not introduce any local or spanwise interference in the incoming undisturbed boundary layer that may influence the flow development in any way.

Relative to no control, significant changes are introduced in the flow field when control devices are implemented. Both RV and RRV devices are seen to introduce large-scale flow structures in the boundary layer compared to no control, Figs. 13 and 14, the growth and scale of which are seen to vary with device height, Fig. 14b, c, and device configuration. The former observation is in conformity with the results of Lee at al. [37] who reported that the size and strength of the vortices shed from a control device increases with increase in device height. Compared to the RV2 (equivalent height) and RV4 (double height) device, the RRV2 device is seen to generate structures of much larger scale immediately downstream of the device the size of which are seen to increase significantly with increase in downstream distance, resulting in a much 


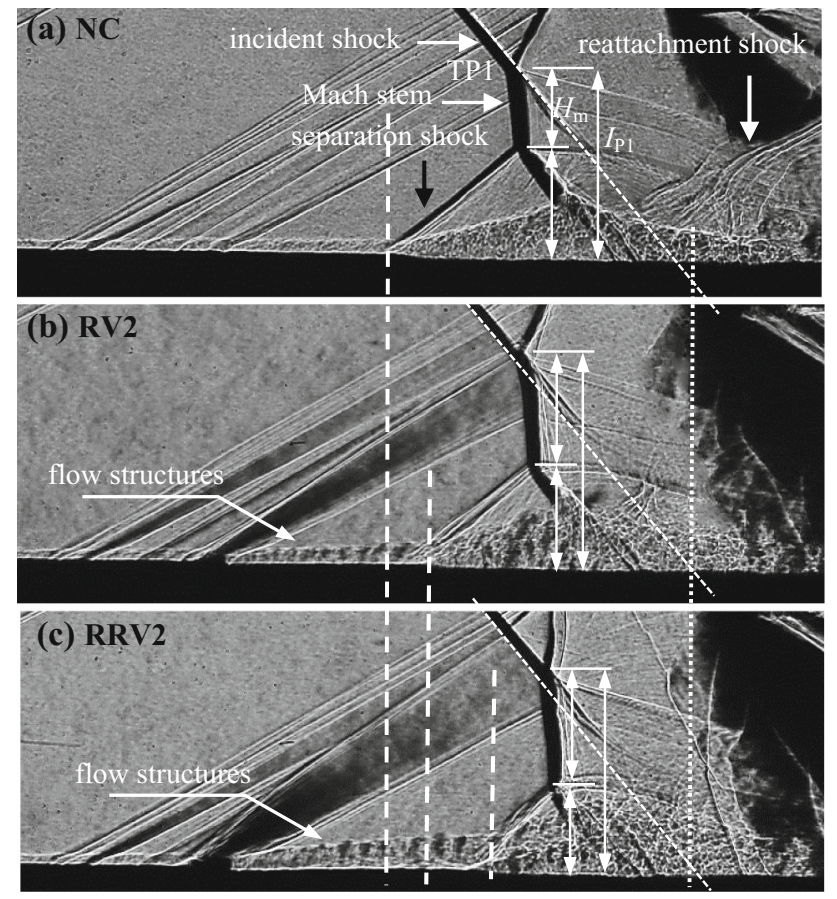

Fig. 13 Schlieren images showing the effect of VGs on the overall flow structure associated with Mach reflection a no control, b RV2, and c RRV $2 ; g / w=0.725$

thicker boundary layer immediately upstream of separation. With regard to this observation, the results of Lee et al. [37] also suggest that the relatively larger-scale structures shed from RRV are also of relatively higher strength compared to those shed from RV3 and RV4 devices. An immediate consequence of this is seen in the form of more effective control capabilities exhibited by RRV2 compared to the RV4 device. Both RV4 and RRV2 devices are seen to push the separation shock location considerably downstream compared to nocontrol case for all $g / w$ cases except for $g / w$ of 0.725 , for which the separation moves significantly upstream for RV3 and RV4 devices, as discussed earlier (figures not shown). Further, important details such as the separation location and Mach stem height are marked in these images with dashed vertical lines and double-headed arrows for an immediate qualitative comparison. These details are then carefully measured ( $\pm 2-3 \%$ accuracy) using MATLAB-based program and plotted in Fig. 15 for an overall comparison. It is clearly seen that the Mach stem and the upper triple point heights $\left(I_{\mathrm{P} 1} / w\right)$ steadily increase with increase in height of $\mathrm{RV}$ device. Although this variation is small in going from $g / w$ of $0.87-0.81$, the difference becomes significantly larger for $g / w$ of 0.725 primarily due to the inherently stronger interaction that this $g / w$ value results in for this experimental setup, as discussed earlier. No significant changes are introduced by RRV devices in the height of Mach stem, although some reductions in the height of TP1 are indicated especially for RRV2.
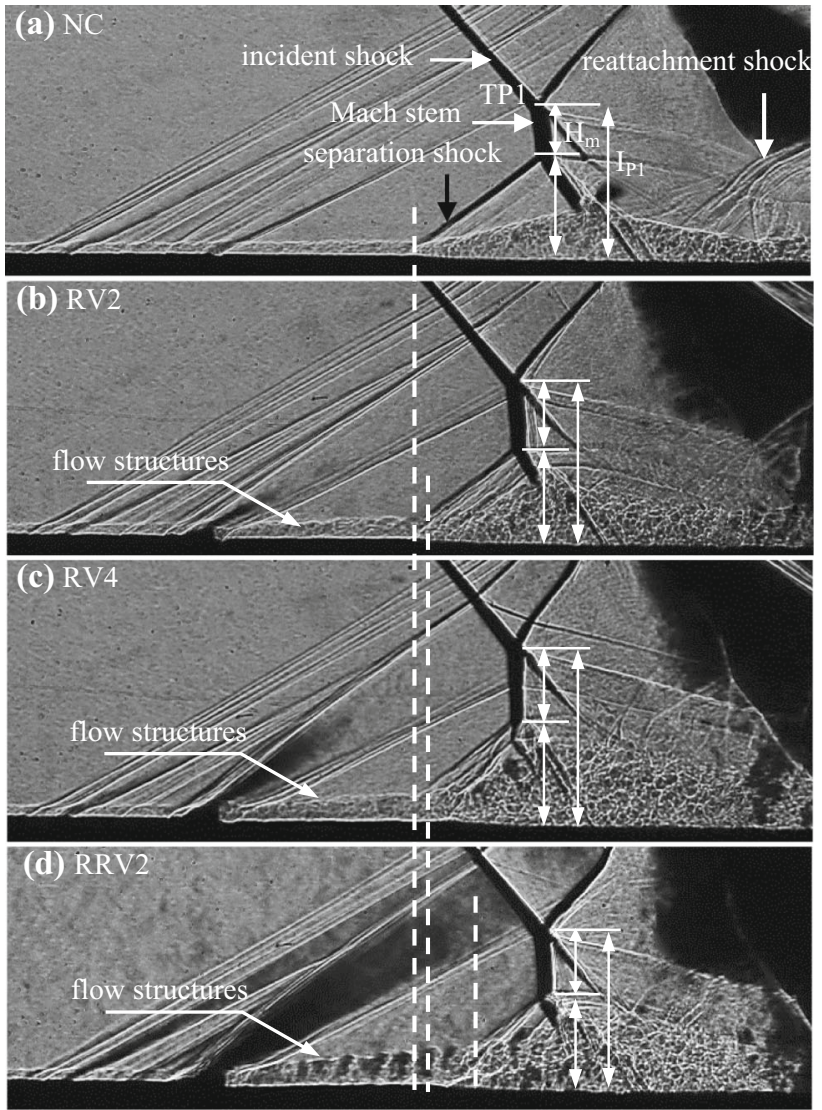

Fig. 14 Schlieren images showing the effect of VGs on the overall flow structure associated with Mach reflection: a no control, b RV2, c RV4, and d RRV2; $g / w=0.81$

It is clear then that (i) the RRV2 device is the most effective in controlling the overall extent of separation in a MR condition irrespective of the $g / w$ value tested and (ii) that the effective control of separation, however, is accompanied with an increase in height of the Mach stem. With regard to the latter, although an increase in the device height is seen to increase the effectiveness of a control device in controlling separation, the advantage is, however, offset by an increase in Mach stem height which would be accompanied with an increase in total pressure losses. However, reducing the separation bubble length and its height with effective control in strong interactions such as these in itself is advantageous in preventing or averting the intake unstart condition at offdesign operation. As a result, a trade-off has to be reached wherein a taller VG is used only for short time off-design condition with reduced inlet performance.

\section{Conclusions}

An experimental investigation was conducted to control a Mach reflection-induced interaction in a Mach 2.05 flow 

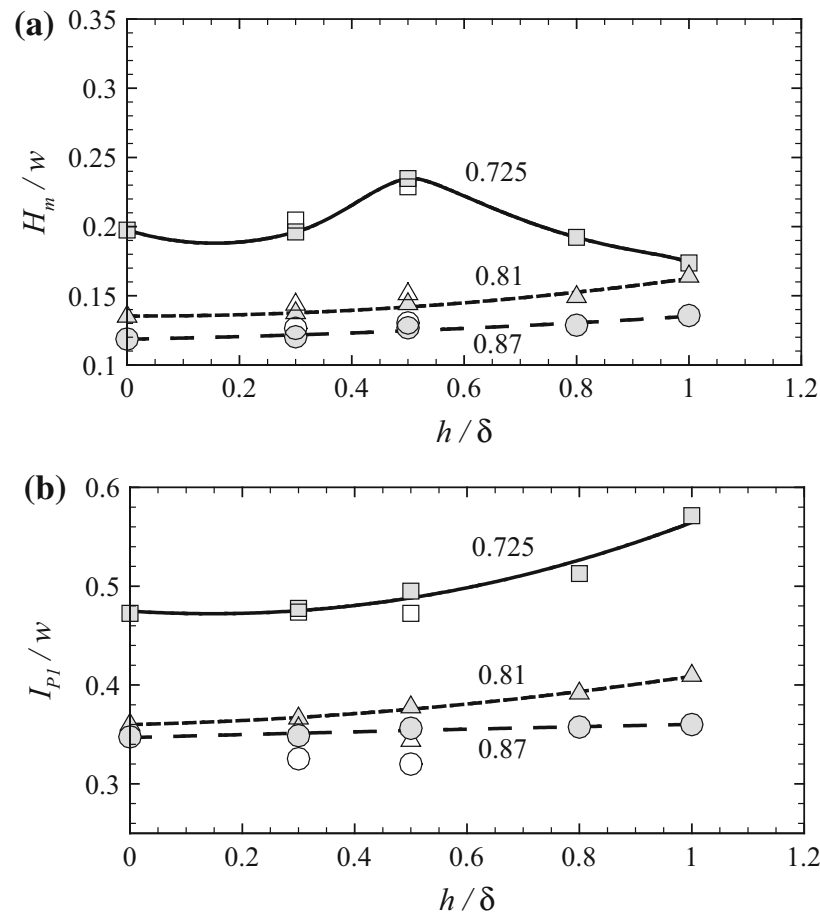

Fig. 15 Variation in a the Mach stem height and $\mathbf{b}$ triple point TP1 height as a function of $g / w$ and control device height

using a $18^{\circ} \mathrm{SG}$. The study was independently conducted for four SG exit heights $(g / w)$ of $0.87,0.81,0.725$, and 0.66 primarily to study its effect on the extent of flow separation as well as on Mach stem height, with and without control. The intention was to initiate and control an intake unstart like conditions wherein the extent of the separation bubble begins to increase with the separation point moving rapidly forward. For this purpose, two vane-type vortex generator configurations, namely the ramp vane with device heights $h / \delta=0.3,0.5,0.8$, and 1.0 and the rectangular vane with $h / \delta=0.3$ and 0.5 , were used for control. Each control device array was implemented $10 \delta$ upstream of the separation location for each $g / w$ no-control case. A total of 25 mean pressure ports and 13 Kulite pressure transducer locations for unsteady pressure measurements were used to study the interaction. Off-surface visualization of the interaction is done using a Z-type schlieren setup, while the surface flow topology is studied using the conventional surface oil visualization technique.

The assessment of no-control mean pressure data shows that while the strength of both the separation and reattachment shocks remains almost similar for SG exit height $g / w$ of 0.87 and 0.81 , a significant increase in these values occurs as the $g / w$ value is further decreased to 0.725 and finally to 0.66 . Similar observations can be made for variation in $\Delta S / w$ and $H_{\mathrm{m}} / w$ values with decreasing $g / w$. This indicates an increasingly stronger interaction for $g / w$ values of
0.725 and 0.66 which is responsible for the observed significant increase in the extent of separation. Schlieren images also show that the MR studied in the present tests could be identified as stable for $g / w=0.87$ and 0.81 and unstable MRs for $g / w=0.725$ and 0.66 . For stable MR interactions, the extent of separation and the strength of the reattachment shock are seen to decrease with increase in RV height with RV4 showing maximum reductions of 17 and $23 \%$ relative to no control, respectively. However, for the unstable MR condition $(g / w$ of 0.725$)$, RV devices of $h / \delta=0.8$ and 1.0 become completely ineffective. The RRV2 device $(h / \delta=0.5)$, on the other hand, was found to be effective in reducing the extent of separation in both the stable $(31 \%)$ and unstable (23\%) MR conditions. A reduction in reattachment shock strength for RRV2 is observed to be approximately $20 \%$ for stable and 35\% for unstable MR. However, the effectiveness of each device configuration to control separation is also seen to be accompanied with an associated increase in the height of the Mach stem. But this is not seen as a serious limitation considering the fact that in strong interactions such as, these it is more important to prevent or avert an intake unstart condition.

The peak rms value, which is a measure of the shock unsteadiness in the interaction region, is initially seen to increase significantly for RV1, relative to no control, and thereafter shows almost similar values independent of RV device height. This trend remains the same with all $g / w$ values, although a decrease in the peak rms values is seen with an increase in $g / w$ value. Although the use of RRV devices brings down the peak rms values significantly compared to an RV device of similar height, the values are still higher than that observed for each no-control case.

Acknowledgements The authors wish to thank the National Trisonic Aerodynamic Facility Division of NAL for their support in the execution of this project. The technical support of Narayana during the model design and fabrication as well as of Janardhan and Jones Philip, staff of the $0.3-\mathrm{m}$ wind tunnel facility at NAL during the test campaigns, is gratefully acknowledged. Special thanks to Gangadhar, Shanmogan, Charan Singh, and Anupam Mantry of the NAL Belur Model Shop for model fabrication.

\section{References}

1. Babinsky, H., Harvey, J.K.: Shock Wave-Boundary-Layer Interactions. Cambridge University Press, Cambridge (2011)

2. Dolling, D.S., Murphy, M.T.: Unsteadiness of the separation shock wave structure in a supersonic compression ramp flow-field. AIAA J. 21, 1628-1634 (1983). https://doi.org/10.2514/3.60163

3. Humble, R.A., Scarano, F., van Oudheusden, B.W.: Unsteady aspects of an incident shock wave/turbulent boundary layer interaction. J. Fluid Mech. 635, 47-74 (2009). https://doi.org/10.1017/ S0022112009007630

4. Délery, J., Dussauge, J.P.: Some physical aspects of shockwave/boundary layer interactions. Shock Waves 19, 453-468 (2009). https://doi.org/10.1007/s00193-009-0220-z 
5. Raghunathan, S.: Passive control of shock boundary layer interaction. Prog. Aerosp. Sci. 25(3), 271-296 (1988). https://doi.org/10. 1016/0376-0421(88)90002-4

6. Stanewsky, E.: Aerodynamic benefits of adaptive wing technology. Aerosp. Sci. Technol. 4, 439-452 (2000). https://doi.org/10.1016/ S1270-9638(00)01069-5

7. Zhang, Y., Tan, H., Tian, F.-C., Zhuang, Y.: Control of incident shock/boundary-layer interaction by a two-dimensional bump. AIAA J. (2014). https://doi.org/10.2514/1.J052786

8. Wong, W.S., Qina, N., Sellars, N., Holden, H., Babinsky, H.: A combined experimental and numerical study of flow structures over three-dimensional shock control bumps. Aerosp. Sci. Technol. 12, 436-447 (2008). https://doi.org/10.1016/j.ast.2007.10.011

9. König, B., Pätzold, M., Lutz, T., Krämer, E., Rosemann, H., Richter, K., Uhlemann, H.: Numerical and experimental validation of threedimensional shock control bumps. J. Aircr. (2009). https://doi.org/ $10.2514 / 1.41441$

10. Ogawa, H., Babinsky, H., Pätzold, M., Lutz, T.: Shockwave/boundary-layer interaction control using three-dimensional bumps for transonic wings. AIAA J. (2008). https://doi.org/10. $2514 / 1.32049$

11. Kim, S.D.: Aerodynamic design of a supersonic inlet with a parametric bump. J. Aircr. (2009). https://doi.org/10.2514/1.37416

12. Chinneck, A., Jones, G. C. A, Tracey. C.M.: An interim report on the use of blowing to reduce fall in control effectiveness associated with shock-induced separation at transonic speeds. ARC 1756 FM2231

13. Delery, J.: Shock wave/turbulent boundary layer interaction and its control. Prog. Aerosp. Sci. 22(4), 209-280 (1985). https://doi.org/ 10.1016/0376-0421(85)90001-6

14. Fukuda, M.K., Hingst, W.R., Reshotko, E.: Control of shock wave-boundary layer interaction by bleed in supersonic mixed compression inlets. J. Aircr. 14, 151-156 (1977). https://doi.org/ $10.2514 / 3.58756$

15. Krogmann, P., Stanewsky, E., Thiede, P.: Effects of suction on shock/boundary-layer interaction and shock-induced separation. J. Aircr. 22(1), 37-42 (1985). https://doi.org/10.2514/3.45077

16. Ball, K.O.W., Korkegi, R.H.: An investigation of the effect of suction on hypersonic laminar boundary-layer separation. AIAA J. 6, 239-243 (1968). https://doi.org/10.2514/3.4484

17. McCormick, D.C.: Shock/boundary-layer interaction control with vortex generators and passive cavity. AIAA J. 31(1), 91-96 (1993). https://doi.org/10.2514/3.11323

18. Barter, J.W., Dolling, D.S.: Reduction of fluctuating pressure loads in shock boundary-layer interactions using vortex generators. AIAA J. 10, 1842-1849 (1995). https://doi.org/10.2514/3.12736

19. Ashill, P.R., Fulker, J.L., Hackett, K.C.: Studies of flows induced by sub boundary layer vortex generators (SBVGs). In: 40th AIAA Aerospace Sciences Meeting and Exhibit, AIAA Paper 2002-0968 (2002). https://doi.org/10.2514/6.2002-968

20. Anderson, B.H., Tinapple, J., Surber, L.: Optimal control of shock wave turbulent boundary layer interactions using micro-array actuation. In: 3rd AIAA Flow Control Conference, AIAA Paper 2006-3197 (2006). https://doi.org/10.2514/6.2006-3197

21. Lin, J.C.: Review of research on low-profile vortex generators to control boundary-layer separation. Prog. Aerosp. Sci. 38(4-5), 389-420 (2002). https://doi.org/10.1016/S0376-0421(02)00010-6

22. Panaras, A.G., Lu, F.K.: Micro-vortex generators for shock wave/boundary layer interactions. Prog. Aerosp. Sci. 74, 16-47 (2015). https://doi.org/10.1016/j.paerosci.2014.12.006

23. Seifert, A., Pack, L.G.: Active control of separated flows on generic configurations at high Reynolds numbers. In: 30th Fluid Dynamics Conference, AIAA Paper 99-3403. https://doi.org/10.2514/6. 1999-3403

24. McCormick, D.C.: Boundary layer separation control with directed synthetic jet. In: 38th Aerospace Sciences Meeting and Exhibit, AIAA Paper 00-0519 (2000). https://doi.org/10.2514/6.2000-519
25. Glezer, A., Amitay, M.: Synthetic jets. Annu. Rev. Fluid Mech. 34, 503-529 (2002). https://doi.org/10.1146/annurev.fluid.34.090501. 094913

26. Narayanaswamy, V., Raja, L.L., Clemens, N.T.: Characterization of a high-frequency pulsed-plasma jet actuator for supersonic flow control. AIAA J. 48(2), 297-305 (2010). https://doi.org/10.2514/ 1.41352

27. Greene, B.R., Clemens, N.T., Magari, P., Micka, D.: Control of mean separation in shock boundary layer interaction using pulsed plasma jets. Shock Waves (2015). https://doi.org/10.1007/ s00193-014-0524-5

28. Cattafesta III, L.N., Sheplak, M.: Actuators for active flow control. Annu. Rev. Fluid Mech. 43, 247-272 (2011). https://doi.org/10. 1146/annurev-fluid-122109-160634

29. Szwabe, R.: Shock wave induced separation control by streamwise vortices. J. Therm. Sci. 14(3), 249-253 (2005). https://doi.org/10. 1007/s11630-005-0009-z

30. Souverein, L.J., Debiève, J.F.: Effect of air jet vortex generators on a shock wave boundary layer interaction. Exp. Fluids 49, 1053 1064 (2010). https://doi.org/10.1007/s00348-010-0854-8

31. Verma, S.B., Manisankar, C.: Shockwave/boundary-layer interaction control on a compression ramp using steady micro jets. AIAA J. 50(12), 2753-2764 (2012). https://doi.org/10.2514/1.J051577

32. Uzun, A., Solomon, J.T., Foster, C.H., Oates, W.S., Hussaini, M.Y., Alvi, F.S.: Flow physics of a pulsed micro-jet actuator for highspeed flow control. AIAA J. 51(12), 2894-2918 (2013). https:// doi.org/10.2514/1.J052525

33. Lin, J.C.: Control of turbulent boundary-layer separation using micro-vortex generators. In: 30th AIAA Fluid Dynamics Conference, AIAA Paper 99-3404, Norfolk, VA, June 28-July 1 (1999). https://doi.org/10.2514/6.1999-3404

34. Jenkins, L., Gorton, S.A., Anders, S.: Flow control device evaluation for an internal flow with an adverse pressure gradient. In: 40th AIAA Aerospace Sciences Meeting and Exhibit, Reno, NV, January 14-17, AIAA Paper 2002-0266 (2002). https://doi.org/10. 2514/6.2002-266

35. Ashill, P.R., Fulker, J.L., Hackett, K.C.: Research at DERA on sub boundary layer vortex generators (SBVGs). In: 39th AIAA Aerospace Sciences Meeting and Exhibit, Reno, NV, January 8-11, AIAA Paper 2001-0887 (2001). https://doi.org/10.2514/6. 2001-887

36. Lee, S., Loth, E., Babinsky, H.: Normal shock boundary layer control with various vortex generator geometries. Comput. Fluids 49(1), 233-246 (2011). https://doi.org/10.1016/j.compfluid.2011. 06.003

37. Lee, S., Loth, E.: Impact of ramped vanes on normal shock boundary-layer interaction. AIAA J. 50(10), 2069-2079 (2012). https://doi.org/10.2514/1.J051253

38. Verma, S.B., Manisankar, C.: Assessment of various low-profile mechanical vortex generators in controlling a shock-induced separation. AIAA J. 55(7), 2228-2240 (2017). https://doi.org/10.2514/ 1.J055446

39. Titchener, N., Babinsky, H., Loth, E.: The effects of various vortex generator configurations on a normal shock wave/boundary layer interaction. In: 51st AIAA Aerospace Sciences Meeting including the New Horizons Forum and Aerospace Exposition, AIAA Paper 2013-0018. https://doi.org/10.2514/6.2013-18

40. Holden, H., Babinsky, H.: Effect of micro vortex generators on separated normal shock/boundary layer interactions. J. Aircr. 44(1), 170-174 (2007). https://doi.org/10.2514/1.22770

41. Verma, S.B., Hadjadj, A.: Supersonic flow control. Shock Waves 25(5), 443-449 (2015). https://doi.org/10.1007/ s00193-015-0587-y

42. Blinde, P.L., Humble, R.A., Oudheusden, B.W., Scarano, F.: Effects of micro-ramps on a shockwave/turbulent boundary layer interac- 
tion. Shock Waves 19, 507-520 (2009). https://doi.org/10.1007/ s00193-009-0231-9

43. Kusunose, K., Yu, N.J.: Vortex generator installation drag on an airplane near its cruise condition. J. Aircr. 40(6), 1145-1151 (2003). https://doi.org/10.2514/2.7203

44. Babinsky, H., Li, Y., Pitt Ford, C.W.: Microramp control of supersonic oblique shock-wave/boundary-layer interactions. AIAA J. 47(3), 668-675 (2009). https://doi.org/10.2514/1.38022

45. Giepman, R.H.M., Schrijer, F.F.J., van Oudheusden, B.W.: Flow control of an oblique shock wave reflection with micro-ramp vortex generators: Effects of location and size. Phys. Fluids 26(6), 066101 (2014). https://doi.org/10.1063/1.4881941

46. Nolan, W.R., Babinsky, H.: Comparison of micro-vortex generators in supersonic flows. In: 6th AIAA Flow Control Conference, AIAA Paper 2012-2812 (2012). https://doi.org/10.2514/6.2012-2812

47. Verma, S.B., Manisankar, C.: Transition control of Mach to regular reflection induced interaction using an array of micro ramp vanetype vortex generators. Phys. Fluids 27(10), 107102 (2015). https:// doi.org/10.1063/1.4932405
48. Skews, B.W.: Aspect ratio effects in wind tunnel studies of shock wave reflection transition. Shock Waves 7(6), 373-383 (1997). https://doi.org/10.1007/s001930050092

49. Van Driest, E.R.: Turbulent boundary layer in compressible flows. J. Aeronaut. Sci. 18(3), 145-160 (1951). https://doi.org/10.2514/ 2.7048

50. O'Donnell, R.M.: Experimental investigation at a Mach number of 2.41 of average skin-friction coefficients and velocity profiles for laminar and turbulent boundary layers and an assessment of probe effects. National Advisory Committee for Aeronautics Technical Note, Report No. NACA TN 3122 (1954)

51. Li, H., Ben-Dor, G.: A parametric study of Mach reflection in steady flows. J. Fluid Mech. 341, 101-125 (1997)

52. Vuillon, J., Zeitoun, D., Ben-Dor, G.: Reconsideration of oblique shock wave reflections in steady flows. Part 2. Numerical investigation. J. Fluid Mech. 301, 37-50 (1995). https://doi.org/10.1017/ S0022112095003788 